\title{
MICROCRÉDito Y DESARROLLO RURAL. UNA MIRADA CRÍTICA A PARTIR DE UN ESTUdIO DE CASO
}

\author{
Microcredit and Rural Development. \\ A Critical View from a Case Study
}

Ayari G. Pasquier Merino ${ }^{1}$

Resumen: En este texto se presentan algunas reflexiones en torno al uso del microcrédito como estrategia en programas de desarrollo rural que buscan combatir las condiciones de pobreza. Con base en la propuesta teórica de Norman Long (2007), se presta particular atención al carácter activo de los actores locales, al papel de las desigualdades y a los procesos de negociación en torno a la operación de este tipo de iniciativas en la esfera local. El trabajo está basado en un estudio de caso elaborado en una pequeńa localidad ubicada en el estado de Chiapas, donde operaba un programa de desarrollo rural estructurado a partir de una serie de proyectos de microcrédito. Con este escenario como trasfondo se analizan los supuestos centrales de la Teoría de la microfinanza y se exponen algunos de los límites que enfrentan los proyectos de microcrédito para superar las condiciones de pobreza y vulnerabilidad de las poblaciones a las que están dirigidos.

Palabras clave: microfinanza, negociación, poder y desigualdad.

\footnotetext{
${ }^{1}$ Doctora en Ciencias Sociales con especialidad en Sociología por El Colegio de México. Posdoctorante en Instituto de Investigaciones Antropológicas de la UNAM. "Estancia posdoctoral en el Instituto de Investigaciones Antropológicas de la Universidad Nacional Autónoma de México. Programa de Becas Posdoctorales en la UNAM". Temas de especialización: desarrollo rural, seguridad alimentaria, gestión colectiva de recursos y migración.

Correo electrónico: ayaripasquier@gmail.com /apasquier@colmex.mx.

Fecha de recepción: 0309 15; Fecha de aceptación: 111215.
}

(cc) EY-NC-ND Páginas 159-189. 
Abstract: In this paper I present some arguments on the use of microcredits as a strategy in rural development programs that aim to combat poverty. Following the theoretical proposal of Norman Long (2007), particular attention is given to the active nature of local actors, the role of inequality and the process of negotiation over the operation of such initiatives at the local level. This work is based on a case study developed in a small town in the state of Chiapas, where a rural development program structured around a series of microcredit projects operated. This scenario serves as background to analyse microfinance theory central assumptions and to expose some of the limits of microcredit initiatives to overcome poverty and vulnerability of rural populations.

Keywords: microfinance, negotiation, power and inequality.

\section{Introducción}

En este texto se presentan algunas reflexiones en torno al uso del microcrédito como estrategia en programas de desarrollo rural que buscan combatir las condiciones de pobreza. Con base en un estudio de caso se analizan los supuestos centrales de la Teoría de la microfinanza y se exponen algunos de los límites que enfrentan los proyectos de microcrédito para superar las condiciones de pobreza y vulnerabilidad de las poblaciones a las que están dirigidos. Al mismo tiempo, se busca mostrar el carácter activo de los actores locales, documentando los procesos a través de los cuales las prácticas financieras que estructuraban una iniciativa de desarrollo rural fueron negociadas y reconfiguradas en su operación cotidiana en las esferas locales, incorporándose en el repertorio de posibilidades de los integrantes de la comunidad.

Las reflexiones que se exponen son resultado de un estudio de caso realizado en Nuevo Porvenir, una pequeña localidad del municipio Las Margaritas ubicada en la parte baja de la zona conocida como "Las Cañadas". En esta localidad operó por tres ańos una iniciativa de desarrollo promovida por una asociación civil llamada Kampo, que buscaba impulsar la producción agrícola comercial a través de diversos esquemas de crédito: préstamos sin intereses, proyectos de microcrédito y un 
crédito para comprar tierras, a un plazo de 10 años. La comunidad estaba integrada por 25 familias — poco más de 150 personas - con derechos de uso sobre un predio de 80 hectáreas. En su mayoría se trataba de familias originarias de Guatemala que llegaron a México durante la década de 1980 como refugiados. Aunque existían diferencias entre ellas respecto a sus recursos e ingresos, todas vivían en condiciones de pobreza extrema y sus modos de sustento dependían del jornaleo agrícola en los potreros y las fincas de café de la región, de las migraciones temporales de los hombres y de las transferencias gubernamentales que recibían las mujeres.

El desarrollo de este trabajo estuvo guiado por la propuesta de Norman Long, quien articula un esquema analítico orientado al actor para estudiar, desde un enfoque etnográfico, las interacciones entre los actores involucrados en los procesos de desarrollo. Desde esta perspectiva las "intervenciones planeadas" son pensadas como "un proceso en movimiento, socialmente construido, negociado, experiencial y creador de significados, no simplemente la ejecución de un plan de acción ya especificado con resultados de comportamiento esperados" (Long, 2007: 65).

El texto está organizado en cinco apartados: en el primero se presentan los principales debates en torno a la microfinanza como herramienta para el desarrollo rural; en el segundo se describe el programa presente en la comunidad de estudio y se analizan algunos aspectos de su operación cotidiana; en el tercero se busca dar cuenta de algunos de los límites de la Teoría de la microfinanza para explicar el funcionamiento de los proyectos de microcrédito; en el cuarto se tratan los temas del capital social y la agencia de los actores locales en los programas de microcrédito; y en el quinto se discuten las reconfiguraciones de los proyectos de microcrédito en las esferas locales.

Concluye el atículo con algunas reflexiones finales en torno a los límites que enfrenta el microcrédito como herramienta para el desarrollo rural. 


\section{Argumentos centrales de la Teoría de la microfinanza como estrategia de desarrollo rural y posiciones críticas}

El microcrédito comenzó a ser difundido como una estrategia alternativa a la inversión estatal en el sector rural de pequeña escala en la década de 1980. Esta estrategia fue promovida en el contexto de la aplicación de las políticas de ajuste estructural que, para el sector agrícola de México, se tradujeron en el desmantelamiento de gran parte de los organismos gubernamentales vinculados con la producción y comercialización agrícola, incluyendo las empresas públicas de crédito y seguros agrícolas (Salas et al., 2010). Diez años después el microcrédito se había convertido en una de las principales intervenciones financieras en el sector rural, y en uno de los pilares de las intervenciones para el desarrollo y la reducción de la pobreza en estos contextos. En algunos países la difusión del microcrédito ha sido fomentada directamente por los gobiernos, pero su expansión responde sobre todo a la acción de organizaciones no gubernamentales y a la multiplicación de instituciones comerciales de microfinanza.

La difusión de este tipo de estrategias presupone que la pobreza es resultado de la exclusión de los servicios financieros formales, situación que orillaría a los pobres a hacer uso de fuentes informales de préstamo, perpetuando así relaciones de poder y dependencia. Desde esta perspectiva la microfinanza tendría el potencial para superar las condiciones de pobreza, dando a los pobres acceso a fuentes de crédito formal — confiable y a precios accesibles - para invertir en infraestructura e insumos productivos, crear pequeñas empresas, diversificar sus estrategias de sustento, estabilizar sus ingresos, disminuir su vulnerabilidad, y mejorar su seguridad alimentaria y capital humano; impulsando así procesos de empoderamiento económico y social (Sebstad y Cohen, 2000; Armendáriz y Morduch, 2005; Chemin, 2008; Bouquet et al., 2009; Collins et al., 2011). Estos postulados exponen de manera sintética los argumentos centrales de la Teoría de la microfinanza, en torno a la cual se ha desarrollado la mayor parte de la literatura sobre microcrédito, y de la cual se han tomado los argumentos para difundir el microcrédito en ámbitos nacionales e internacionales y diseñar sus estrategias de intervención. 
Sin embargo, los efectos de la microfinanza en la pobreza y vulnerabilidad de las familias rurales es un tema cada vez más controvertido. La literatura muestra que distintas relaciones de deuda pueden proteger o arriesgar la estabilidad económica de un hogar, llevar al empobrecimiento o contribuir a la acumulación de capital, crear o reforzar jerarquías, o confrontarlas, así como mantener o erosionar las relaciones de solidaridad (Zelizer, 1994; Montgomery, 1996; Villarreal, 2004; Guérin et al., 2009; Islam, 2015).

Los presupuestos en los que se basa el desarrollo de la Teoría de la microfinanza coinciden con un supuesto general que considera la deuda como un recurso que favorece el crecimiento económico, pero ha sido ampliamente documentado que las condiciones que enfrentan los hogares en pobreza imponen una alta exposición al riesgo, por lo que el endeudamiento incrementa su vulnerabilidad (González de la Rocha, 2006; Long, 2007; Villarreal, 2004, 2010; Guérin, 2011). Las incertidumbres que enfrentan las actividades agrícolas incrementan esta vulnerabilidad, sobre todo cuando se trata de proyectos de microcrédito con plazos muy cortos (Duvendack et al., 2011). Por otra parte, los riesgos vinculados con las iniciativas de microcrédito afectan sobre todo a las familias más pobres, pues tienen poca capacidad de inversión, es más probable que necesiten usar los créditos para el consumo, si tienen algún problema corren el riesgo de tener que vender los pocos activos que poseen y, al tener menor poder de negociación, suelen ser objeto con mayor frecuencia de las sanciones previstas por el incumplimiento de los pagos (Montgomery, 1996; Mosley y Hulme, 1998; Mosley, 2001; Shaw, 2004; Guérin et al., 2009; Guérin, 2011).

Un creciente número de estudios ha documentado casos donde el aumento de la oferta de microcrédito rural dirigido a pequeños productores ha contribuido al empobrecimiento de sus "beneficiarios", creando nuevas "trampas de pobreza" (Guérin et al., 2009; MorvantRoux, 2009; Guérin et al., 2011; Bateman, 2012; Harper, 2012; Marr, 2012; Bateman y Skare, 2014), ha incrementado la desigualdad (Taylor, 2012) y provocado conflictos dentro de las comunidades (Marr, 2003; Bateman y Chang 2009). 
También se ha señalado que la Teoría de la microfinanza fue desarrollada a partir de análisis teóricos, mientras que la mayor parte de los estudios empíricos elaborados en este marco se limitaron a medir el "éxito" de las iniciativas con base en su sostenibilidad financiera, dando por hecho su impacto positivo para los hogares rurales sin presentar evidencia empírica contundente que sostuviera estas afirmaciones (Fouillet y Augsburg, 2008; Duvendack et al., 2011; Bateman, 2012; Rajbanshi et al., 2015).

En este artículo se discuten algunos de los postulados centrales de la Teoría de la microfinanza con base en el estudio de caso realizado en Nuevo Porvenir. Este trabajo se suma así al creciente número de estudios que cuestionan los supuestos teóricos de la Teoría de la microfinanza y contradicen su efectividad como estrategia de desarrollo rural.

\section{Un estudio de caso sobre la operación de proyectos de micro- crédito para la producción agrícola: la operación de Kampo en Nuevo Porvenir}

La iniciativa de desarrollo que operaba en Nuevo Porvenir compartía los postulados generales de la Teoría de la microfinanza pero, a diferencia de la mayor parte de las iniciativas de microfinanza, Kampo ofrecía créditos individuales por montos variables y con plazos amplios, dando derechos de uso sobre un terreno que, según su modelo operativo, debía servir como activo de base para el pago de las cuotas.

El modelo de intervención de esta asociación estaba enfocado en el impulso de la producción agrícola: el cultivo comercial de café y plátano $\mathrm{y}$, de manera marginal, pequeñas actividades familiares como hortalizas, cría de aves de corral o cerdos, negocios de abarrotes, etc. Según la narrativa del programa estas actividades proporcionarían a los miembros de la comunidad los ingresos para pagar los créditos dirigidos al desarrollo de actividades económicas y las cuotas por el terreno que les fue puesto a disposición, con el fin de superar en el corto plazo su condición de pobreza.

Estas actividades eran financiadas a través de distintos esquemas de microcrédito: la mayoría de los créditos financiaban el desarrollo de iniciativas productivas ya establecidas y manejaban una tasa de interés de 
$10 \%$ anual y plazos de entre 6 y 12 meses; de manera complementaria se ofrecían préstamos para promover proyectos de carácter experimental, cuyo rembolso estaba sujeto a las ganancias efectivamente obtenidas.

El programa de Kampo representaba una fuente de crédito relativamente accesible para el desarrollo de actividades productivas y la capitalización de las familias más pobres en la región, llenando un vacío evidente en el contexto local. Sin embargo, esto no quiere decir que la iniciativa de Kampo operara en el vacío. El "panorama financiero" local incluía una multiplicidad de prácticas informales de crédito: pequeños préstamos sin intereses a corto plazo entre personas cercanas, venta de insumos cotidianos con pagos diferidos, venta por pagos escalonados, empeño de pequeños bienes y préstamos de sumas más importantes con tasas de interés de entre 15 y $20 \%$ mensual. Estas prácticas coinciden con lo reportado en otros estudios que documentan las prácticas financieras en contextos rurales en México (Villarreal, 2000; Angulo, 2004). En caso de necesidad los integrantes de Nuevo Porvenir también recurrían a quienes los contrataban como jornaleros para solicitar préstamos a cambio de trabajo, acuerdos similares eran utilizados para adquirir insumos agrícolas.

Los integrantes de Nuevo Porvenir tenían una apreciación positiva sobre los créditos que les ofrecía Kampo, pues representaban una oportunidad poco frecuente para desarrollar actividades productivas, aunque algunos de ellos sabían por experiencias previas que las iniciativas impulsadas enfrentaban múltiples obstáculos, se mostraban escépticos y se quejaban de la falta de seguimiento a los proyectos. El personal operativo de la asociación buscaba tranquilizar a quienes les presentaban sus dudas, argumentando que era suficiente trabajar bien para no tener problemas, pero a pesar de los grandes esfuerzos de las familias se haría cada vez más evidente que no era fácil ajustarse a los planes de trabajo establecidos para cumplir con las expectativas del programa. Esta situación daría pie a una serie de reajustes y negociaciones de los pactos inicialmente establecidos entre los integrantes de Nuevo Porvenir y el personal de Kampo.

A partir del análisis de la práctica cotidiana de este programa se sugiere que la operación de las estrategias de intervención en esferas 
locales no puede ser comprendida como la simple traducción de los modelos que las inspiran en acciones predefinidas que el personal de las agencias impulsa para involucrar a sus beneficiarios. Su operación pasa por distintos momentos de reinterpretación en los que los operadores del programa y sus beneficiarios negocian según sus posibilidades e intereses. Este planteamiento coincide con la identificación de espacios de "interfaz social", concepto con el que Norman Long (2007) identifica aquellos espacios de interacción continuada que ocurren en los puntos donde se cruzan diferentes mundos sociales, con distintos intereses, interpretaciones, conocimientos, relaciones y poderes, donde puede observarse cómo operan las discrepancias y qué factores resultan relevantes en la distribución de los recursos que se dan a través de estas relaciones. El autor plantea el uso de este concepto como una alternativa a la de "participación local", pues le permite deconstruir el concepto de "intervención planeada", mostrando estas iniciativas como procesos de negociación, adaptación y transformación que tienen lugar entre actores con conocimientos y poderes desiguales. Estos argumentos resultan particularmente relevantes para comprender la gestión y manejo de los proyectos de microcrédito en el estudio de caso desarrollado en Nuevo Porvenir.

Según las reglas operativas de Kampo, el procedimiento para obtener un crédito iniciaba en las reuniones de planeación anual organizadas por el personal operativo de la asociación, encargado de definir un plan de créditos según los intereses que expresaban los integrantes de la comunidad y los recursos que la directiva de la asociación asignara a las diferentes áreas. En la práctica, este esquema de planeación era difícil de operar pues los productores se mostraban reticentes a establecer un calendario anual de créditos de manera colectiva y preferían acercarse de manera individual al personal de la asociación cuando enfrentaban necesidades específicas — por ejemplo, fertilizar sus plantas de café cuando las veían muy cargadas.

Además, aun si la normativa de la asociación señalaba que los recursos debían ser distribuidos con base en la historia crediticia de cada familia y sus potenciales productivos, la aprobación de los créditos productivos era discrecional y dependía en gran medida de su función en el manejo 
de las relaciones del equipo de la asociación con el conjunto de los titulares de derecho. Esto procesos sugieren que la obtención y gestión de un crédito y otros recursos que pasan a través de una organización no gubernamental son procesos de negociación continua, de acomodos y compromisos, y muchas veces son fuente de tensiones o conflictos abiertos (Guérin, 2011).

Para la gestión y el seguimiento de los proyectos de crédito el modelo de Kampo preveía la organización de un comité ad hoc, integrado por tres titulares de derecho de la comunidad. Al inicio de mi investigación el responsable de este comité me explicó sus funciones en los siguientes términos: "Cuando alguien quiere un crédito se acerca con nosotros, y nosotros tenemos que avalar los créditos que los compañeros quieran pedir, solicitamos el crédito con los ingenieros, hacemos el trámite, repartimos el dinero y también tenemos que cobrar cuando llega la fecha que toca el pago para dárselo a los de Kampo".

Algunos meses después, el coordinador regional de la asociación me explicó que este comité había sido formado sólo para la gestión de los créditos de producción dirigidos a los proyectos en los que participaba el conjunto de los titulares de derecho - las plantaciones de café y plátano-, describiendo sus funciones en términos sustancialmente distintos:

Para aprobar los créditos la oficina nos solicitaba una "justificación de rentabilidad económica”, pero en la comunidad la gente tiene muchas dificultades para calcular la rentabilidad de sus cultivos, o siquiera el tiempo que les llevaría obtener ganancias, esto por sus deficiencias de tipo técnico. Por eso nosotros tratábamos que el comité hiciera eso con los créditos comunitarios, que avalaran los diagnósticos de rentabilidad y respaldara esa justificación para que nos aprobaran los créditos ... En cambio de los créditos pequeños, que eran para proyectos personales, siempre nos ocupamos nosotros.

El equipo de la asociación no estaba interesado en delegar funciones en la gestión de los créditos, pues la interacción individual vinculada con 
el crédito era fundamental para la operación del programa. Como me comentó el coordinador regional de la asociación:

[...] entregando los créditos a cada quien, por individuo, se busca que cada quien reconozca su deuda, y además como en la entrega teníamos su interés era el único momento que estábamos seguros de encontrarlos y poder completar la documentación del registro.

La centralidad del personal de Kampo en la gestión de los créditos coincide con situaciones reportadas en otros estudios sobre las dinámicas sociales en torno al microcrédito, donde se muestra que las relaciones entre los agentes de crédito y sus clientes no se limitan a una relación contractual (Guérin, Fouillet y Palier, 2007; Morvant-Roux y Guérin, 2012).

El programa de Kampo estaba compuesto por distintos esquemas crediticios. Según el modelo de la asociación, aquellos que permitían mayor flexibilidad debían estar dirigidos a financiar actividades económicas experimentales, buscando promover la diversificación económica de la comunidad. Sin embargo, el personal operativo de la asociación privilegiaba en este tipo de iniciativas a las familias más pobres de la comunidad, buscando así beneficiarlas con pequeñas actividades productivas que pudieran mejorar sus ingresos, sin que los riesgos de una nueva deuda des-incentivaran su disposición de emprender nuevos proyectos. Al mismo tiempo, estas prácticas permitían fomentar la cultura del crédito y reinversión de las ganancias, lógica que la asociación buscaba inculcar entre sus beneficiarios. Asimismo, en la asignación de este tipo de proyectos el equipo de Kampo parecía premiar a quienes habían dado mayor respaldo a la asociación y fomentar el cumplimiento de las reglas del programa. Distintos autores (Fouillet y Pairault, 2012; Guérin, 2015) han documentado prácticas similares en la India cuando analizan los esfuerzos de las instituciones de microfinanza para transmitir a sus clientes la "disciplina financiera" que consideran central para el éxito de sus iniciativas. 
En cambio, según me explicó el coordinador regional de la asociación, la aprobación de los créditos para la producción en proyectos ya establecidos — con una tasa de interés de 10\% anual- dependía de la capacidad de pago de los titulares de derecho. Esto coincide con una tendencia general entre las instituciones de microfinanza de mejorar la sostenibilidad económica a expensas de los objetivos sociales de dichos programas. Esta tendencia ha favorecido a quienes se ajustan a los productos crediticios disponibles (Rankin y Shakya, 2012; Helth y Dahl, 2014; Labie et al., 2015), contradiciendo la compatibilidad de los objetivos económicos y sociales, uno de los presupuestos centrales en la difusión de la microfinanza.

Llama la atención que este tipo de créditos fueron dados en todos los casos a personas con una posición de poder dentro de la comunidad. Si bien es cierto que estas familias tenían mayor capacidad de endeudamiento, debe tenerse en cuenta que, dada su posición de liderazgo, tenían un papel central en la relación entre el personal operativo de Kampo y la comunidad, pues su intervención podía modificar las capacidades para construir acuerdos favorables al programa. En este sentido se podría decir que la aprobación de créditos para la producción era parte de una lógica más amplia de "intercambio de favores" que buscaba facilitar la operación del programa.

Por otra parte, también vale la pena mencionar que en ocasiones las áreas en las que Kampo buscaba fomentar el crédito no coincidían con los intereses de los integrantes de Nuevo Porvenir. Esta situación podía dar pie a largas negociaciones que muchas veces no llegaban a nada y los productores terminaban por usar los créditos para lo que más le convenía. Esta "manipulación” en el uso de los créditos por parte de los integrantes de la comunidad puede ser interpretada como muestra de que el programa de Kampo había sido incorporado entre los recursos y estrategias de los actores locales, no de manera pasiva como preveían las normas de operación del programa, sino transformado según los intereses y estrategias de los participantes y las estructuras de poder de la comunidad.

En el modelo de intervención de Kampo, el microcrédito era planteado como una herramienta central para el desarrollo productivo 
de la comunidad, y sus directivos ejercían cierta presión sobre el personal operativo de la asociación para que impulsaran nuevos proyectos. En la medida en que se fueron acumulando las líneas de crédito, y las deudas de los integrantes de la comunidad, comenzó a vivirse una tensión creciente, de ahí que seguir el esquema planteado por el modelo de la asociación se hizo cada vez más difícil. Los integrantes de la comunidad tenían dificultades para cumplir con los pagos, pedían prorrogas y los esquemas de pago eran continuamente reconfigurados. El crédito era considerado como una oportunidad pero el endeudamiento se vivía como un problema. Con el tiempo las tasas de pago fueron cayendo y los pagos atrasados se acumularon. En estas condiciones los integrantes de la comunidad comenzaron a rehusar el contraer nuevas deudas, mientras que entre el personal operativo de la asociación surgían dudas sobre la efectiva conveniencia de impulsar nuevos proyectos de crédito, pues el decremento de las cuotas de recuperación afectaba también la "disciplina financiera" necesaria para el funcionamiento del programa a mediano y largo plazo.

Las dificultades que enfrentaban los integrantes de Nuevo Porvenir para pagar los créditos estaban relacionadas con la vulnerabilidad de las ganancias que podían obtener de sus cosechas; se veían afectadas por la variabilidad climática, la falta de infraestructura para almacenar y procesar los productos, las dificultades relacionadas con su comercialización y, más en general, por la marginación social y territorial de Nuevo Porvenir. A esto se sumaba la falta de fuentes de empleo en la región. Además, la presión por mantener el consumo de los hogares llevaba a las familias a utilizar las pocas ganancias obtenidas de la producción agrícola para financiar sus gastos cotidianos. En algunos casos el incumplimiento de los pagos también estaba relacionado con la necesidad de cubrir gastos imprevistos y con la precariedad de las condiciones de salud de la población. Situaciones similares han sido reportadas en otros trabajos (Guérin et al., 2011; Bateman, 2012; Taylor, 2012; Marr, 2012; Guérin, 2011 y 2015).

En este sentido vale la pena decir que si bien la iniciativa de Kampo redujo la exclusión de los servicios financieros formales que habían enfrentado los integrantes de Nuevo Porvenir hasta entonces, no les 
permitió superar su dependencia de los préstamos informales, como presupone la teoría de la microfinanza, pues el programa ofrecía únicamente créditos destinados a financiar actividades productivas. Así, los integrantes de Nuevo Porvenir seguían dependiendo de los préstamos usureros ofrecidos en la región por comerciantes y rancheros para cubrir gastos asociados con enfermedades, ceremonias, pérdida de cosechas u otros imprevistos. Esta situación coincide con cuanto ha sido reportado en otros estudios (Guérin, 2015; Islam et al., 2015).

El personal operativo de Kampo era condescendiente frente al retraso de los pagos y buscaba privilegiar los objetivos sociales frente a la sostenibilidad económica del programa, negociando prórrogas para los morosos con la oficina administrativa de la asociación. Éstos retrasos generaban algunos intereses, pero siendo tasas anuales sobre cantidades pequeñas no eran percibidas como un problema por los integrantes de la comunidad. Además, en el contexto local no había instituciones gubernamentales que respaldaran a Kampo para hacer valer sus acuerdos contractuales, mientras la morosidad en los pagos de los créditos del programa era tan difusa que no parecía comportar algún estigma social, condiciones identificadas en otros contextos como definitorias para asegurar el pago de los créditos. El coordinador regional de Kampo intentó consolidar la práctica de pequeños pagos periódicos, argumentando que quienes se esforzaran por cumplir sus compromisos tendrían nuevas líneas de crédito en el futuro. En ausencia de medidas de coacción esta promesa parecía ser el único incentivo para pagar las cuotas pactadas, pero no tuvo el impacto esperado.

La experiencia de Kampo en Nuevo Porvenir diverge respecto a los procesos reportados en muchos contextos rurales, en México y otros países, donde frente a las sanciones sociales e institucionales contra los clientes morosos, y la presencia de múltiples instituciones de microcrédito, la gente se endeuda con diversas instituciones a la vez para pagar las cuotas de compromisos previos y mantener varias líneas de crédito abiertas (Rodríguez y Villarreal, 2014; Schicks, 2014; Angulo, 2014).

El modelo de Kampo parecía operar bajo el presupuesto de que la gente que se compromete en acuerdos crediticios debe pagar sus 
deudas. Sin embargo, éste es un argumento con bases más morales que económicas (Graeber, 2011). En Nuevo Porvenir la convicción de pagar los créditos estaba relacionada con múltiples factores. Las expectativas, obligaciones y compromisos establecidos en el marco del programa jugaban un papel central. Aunque los cálculos y estrategias de los actores locales también estaban influenciados de manera importante por los significados que daban a los recursos provenientes del exterior - que podían ser interpretados como una donación, un derecho o una oportunidad - según su origen y destino (Zelizer, 1997) y las experiencias previas con instituciones de ayuda y desarrollo de los sujetos.

De manera más general, este estudio sugiere que las dinámicas sociales que se dan en torno a una iniciativa de desarrollo tienen mucho que ver con las representaciones e interpretaciones de los actores involucrados, por lo que su estudio no puede confinarse a la delimitación espaciotemporal de un proyecto. Estos argumentos respaldan el planteamiento de Norman Long (2007), quien señala:

[...] conceptuar la intervención como una actividad circunscrita y claramente localizada encubre el importante asunto teórico de que la intervención nunca es un "proyecto" con límites claros en tiempo y espacio, como es definido por el aparato institucional del Estado o por la entidad implementadora. Las intervenciones siempre forman parte de una cadena o flujo de eventos localizados en una estructura más amplia de actividades de los cuerpos estatales y/o internacionales y las acciones de diferentes grupos de interés que operan en la sociedad civil (Long, 2007: 77-78).

[...] del lado del "intervenido", está el conocimiento acumulado de experiencias anteriores de intervenciones de varias clases ... y lo mismo se sostiene en relación con los grupos en instituciones definidos como las "partes interventoras"... - a escala institucional e individual- ... Los procesos de intervención específicos deben ser vistos, 
por consiguiente, en relación con las memorias colectivas e individuales (ibídem: 80).

La investigación también sugiere que el funcionamiento de los proyectos de microcrédito tiene que ver con las relaciones de poder entre los operadores de una iniciativa y los actores locales. En este sentido el estudio de caso documenta cómo los actores locales fueron apropiándose del programa y, en la medida en que comprobaron que el personal de Kampo carecía de mecanismos para hacer efectivos los acuerdos crediticios, impusieron sus propias condiciones. Después de algún tiempo los integrantes de Nuevo Porvenir dejaron de pagar los créditos que habían adquirido con Kampo y la directiva del programa decidió limitar la trasferencia de recursos hacia la comunidad. Como resultado, los integrantes de Nuevo Porvenir perdieron interés en el programa. Al mismo tiempo, surgió un creciente malestar entre ellos por el tiempo que invertían en actividades a las que convocaba el personal operativo de Kampo, sobre todo por la frecuencia de las reuniones. A esto se sumaba una creciente conflictividad dentro de la asamblea y en la relación con el equipo de la asociación. Después de tres años de operación del programa la relación entre el equipo de Kampo y la asamblea comunitaria de Nuevo Porvenir se había hecho cada vez más problemática y, en el marco de un conflicto generalizado, los productores pospusieron los pagos de manera indefinida y el programa suspendió sus operaciones.

Este caso muestra que la obtención y gestión de un microcrédito en el marco de programas de desarrollo representan procesos de negociación continua en los que participan actores con percepciones diferentes y posiciones desiguales, donde muchas veces aparecen tensiones o conflictos abiertos.

\section{El "fracaso" del programa de Kampo en Nuevo Porvenir}

En este apartado se busca dar cuenta de algunos de los límites de la Teoría de la microfinanza para explicar el funcionamiento de los proyectos de desarrollo rural que incluyen estrategias de microcrédito, cuyos 
argumentos pueden servir para explicar situaciones particulares que no son generalizables.

En el marco de la Teoría de la microfinanza se han identificado como explicaciones de la falta de pago de los créditos: el "azar moral" (Stiglitz, 1990), que se refiere a la selección de proyectos de inversión poco exitosos; los altos costos de monitoreo sobre el desarrollo de las inversiones (Banerjee et al., 1994); la falta de mecanismos para aplicar los contratos — sanciones- (Besley y Coate, 1993); y las fallas en la selección de los prestatarios (Ghatak, 1999). Estos estudios coinciden en identificar una relación inversa entre la tasa de interés y las tasas de pago de los créditos, y un efecto positivo de la productividad de las inversiones en las tasas de pago. Joseph Stiglitz (1990) y Abhijit Banerjee et al. (1994) también coinciden en señalar que el monto prestado afecta de manera inversa la tasa de pago. Estos argumentos resultan pertinentes para explicar casos específicos, pero difícilmente pueden ser generalizados (Ahlin y Townsend, 2007).

El "azar moral" no resulta un factor relevante en el caso estudiado, la gran mayoría de los proyectos en los que se invirtieron los créditos eran definidos a priori por Kampo. La relación inversa entre las tasas de interés y las tasas de pago tampoco parecería confirmarse en el caso de Nuevo Porvenir, aun si algunos integrantes de la comunidad expresaban su molestia porque se cobraran intereses a un préstamo que ellos consideraban "de carácter social" —o, en palabras de algunos de los entrevistados, "para ayudar a los pobres"-, las tasas impuestas por Kampo se consideraban como tasas bajas y éste no era un argumento en disputa.

La falta de rentabilidad a corto plazo de las inversiones sí contribuyó de manera importante en el cuestionamiento del programa por parte de los integrantes de la comunidad, y en el desenlace de la relación entre Kampo y los integrantes de Nuevo Porvenir. Las fallas en la selección de los prestatarios constituye también un argumento relevante para el caso de estudio pues, en los hechos, el proceso de selección de los beneficiarios del programa no respondía al perfil definido por el modelo de la asociación para aumentar las posibilidades de éxito de sus proyectos sino a las relaciones del personal operativo de Kampo con la 
asamblea de la comunidad, y en particular con las personas que tenían mayor influencia en la toma de decisiones colectivas.

No se observa una relación inversa entre el monto prestado y las tasas de pago, incluso los créditos que representaban pequeñas cantidades no fueron pagados. El caso estudiado muestra como impedimentos centrales para el cumplimiento de los pagos de este tipo de créditos: la baja productividad de las actividades agropecuarias, los múltiples obstáculos contextuales que enfrentaba su desarrollo, la falta de fuentes alternativas de ingreso y las necesidades cotidianas de las familias.

Los costos de monitoreo no eran relevantes en este caso dado el número reducido de integrantes de la comunidad y su convivencia cotidiana. En cambio, el hecho de que Kampo no contara con mecanismos para asegurar la aplicación de los acuerdos de crédito fue sin duda uno de los elementos fundamentales para explicar las bajas tasas de pago de los créditos a lo largo del programa, y el desenlace de su relación con los titulares de derecho de Nuevo Porvenir. En la medida en que avanzaba el programa los titulares de derecho fueron dándose cuenta de que el equipo de Kampo tenía pocos recursos, más allá de los intentos de conciliación o las sanciones verbales, para hacer cumplir sus normas —incluyendo el pago de los créditos—, esto permitió que se fueran ampliando los espacios para el cuestionamiento de los acuerdos crediticios. Cuando los titulares de derecho decidieron dejar de pagar los créditos, la asociación se encontró prácticamente sin opciones para hacer efectivo sus acuerdos contractuales con los integrantes de la comunidad.

\section{Capital social, agencia y microcrédito}

En la Teoría de la microfinanza hay un supuesto bastante generalizado sobre el efecto positivo del capital social en las tasas de pago. En este sentido se argumenta que cuando existen relaciones estrechas entre los integrantes de un grupo se genera mayor confianza sobre la contribución equitativa de todos los miembros, incentivo que explicaría las altas tasas de pago en los proyectos de crédito colectivo - donde el grupo se hace responsable por el pago de cada uno de sus integrantes- (Cassar et al., 2007). 
En el caso de estudio, los integrantes de Nuevo Porvenir y los operadores de Kampo coincidían en identificar la gestión colectiva de los créditos como conflictiva, pues en las reuniones enfrentaban dificultades para construir acuerdos. Sin embargo ambas partes buscaban beneficiarse de los espacios colectivos para respaldar sus propios intereses.

El programa de Kampo no preveía créditos con esquemas de coresponsabilidad y todos los créditos eran considerados relaciones contractuales de carácter individual, pero algunos aspectos de su gestión eran colectivos: se buscaba acordar un calendario de créditos en reuniones de planeación anual y se preveía la intermediación de la "comisión de créditos" en algunas facetas de la aprobación y cobranza de los créditos individuales. Los líderes de la comunidad, por su parte, argumentaban que existiese un compromiso colectivo por parte de la comunidad para mantener las reglas del programa y asegurar que todos los titulares cumplieran con las cuotas de pago. Sin embargo, cuando algún titular de derecho se retrasaba en los cultivos, comprometiendo sus capacidades futuras de pago, o no cumplía con las cuotas de los créditos que se le habían otorgado, las autoridades podían llamarlo "para platicar", pero nunca impusieron otro tipo de sanción y por lo general buscaban justificar los retrasos de sus compañeros comentando con el equipo de Kampo las difíciles condiciones económicas que enfrentaban las familias — por ejemplo, por la falta de empleo o los precios bajos que habían obtenido por sus cosechas.

La intervención de Kampo se beneficiaba de la organización interna de la comunidad para la operación del programa, como argumentan quienes sostienen que un alto nivel de capital social tiene un impacto positivo en los programas de microcrédito, pero la capacidad de los líderes comunitarios para construir consensos limitaba las posibilidades de que Kampo impusiera sus reglas, aumentando el poder de negociación de la asamblea y sus posibilidades de confrontar el programa.

En el presente caso de estudio el capital social dentro de la comunidad permitió que los miembros de la asamblea ganaran fuerza frente a Kampo y se opusieran en bloque al pago de los créditos, colectivizando sus deudas individuales para no pagar las cuotas. Esta 
situación coincide con los planteamientos de Abhijit Banerjee et al. (1994) y Timothy Besley y Stephen Coate (1995), quienes identifican la cooperación dentro de un grupo, los intercambios frecuentes y los vínculos familiares entre sus miembros como factores que dificultan la aplicación de sanciones, incidiendo de manera negativa en las tasas de pago de los microcréditos. Este tipo de afirmaciones cuestionan los supuestos sobre la efectividad de los colaterales sociales como clave de éxito en los grupos de crédito. Resultados similares han sido documentados por distintos autores (por ejemplo: Griffin y Husted, 2015; y Czura, 2015).

\section{Prácticas trasgresoras y cambio de significados}

Como se ha expuesto, los miembros de Nuevo Porvenir trasgredieron en distintas formas las normas y estrategias propuestas por el programa de Kampo, expresando cierta resistencia ante las lógicas económicas del programa. Inicialmente estas transgresiones se combinaron con formas de conformidad y cooperación, pero ponían constantemente a prueba los límites del reglamento inicialmente impuesto por la asociación. Entre las trasgresiones destaca el uso de las ganancias provenientes de las cosechas para subsidiar el gasto cotidiano, y no para el pago de los créditos; situación común en contextos de pobreza donde se enfrentan grandes carencias e incertidumbres (Rankin y Shakya, 2012). Otras de las transgresiones frecuentes eran no asistir a las reuniones, aun cuando se cobrara una multa por la falta, o asistir sin participar de manera activa.

Katherine Rankin y Yogendra Shakya (2012) reportan hechos similares en un estudio realizado en Vietnam y Nepal, en el que proponen una tipología de "prácticas trasgresoras". Con base en esta tipología, las trasgresiones de los integrantes de Nuevo Porvenir en el marco del programa de Kampo pueden identificarse como prácticas "subversivas", caracterizadas como prácticas trasgresoras que no generan cambios en los programas pero sí modifican los significados que se dan a las intervenciones en la esfera local. En el caso de Nuevo Porvenir puede plantearse que los créditos que el modelo de Kampo había configurado como "tecnologías disciplinarias" para trasmitir a los actores locales una lógica comercial fueron reconfiguradas como 
estrategias para enfrentar las prioridades de corto plazo de familias que vivían en un contexto de múltiples incertidumbres asociadas con sus condiciones de pobreza.

Según se pudo documentar a lo largo de la investigación, las prácticas trasgresoras que ha enfrentado Kampo en diversas comunidades no han tenido efectos en el diseño de su modelo. Las modificaciones realizadas en los últimos años a éste respondían sobre todo a los imperativos económicos de la asociación, que dio cada vez mayor importancia a la sostenibilidad económica de sus proyectos.

Esta investigación también coincide con la posición de Katherine Rankin y Yohendra Shakya (2012) sobre el carácter contradictorio de las prácticas trasgresoras documentadas y la necesidad de ser prudentes al atribuirles una intencionalidad dada, pues aun si pueden ser interpretadas como maneras de cuestionar los modelos de intervención, muchas veces responden sobre todo a los imperativos cotidianos de los actores locales.

Por otra parte, el presente estudio muestra que, en ciertos contextos, el poder de las intervenciones planeadas sobre las comunidades locales puede ser limitado, y su funcionamiento depende de las capacidades del personal de las asociaciones que las promueven para adaptar sus reglas operativas al contexto local, negociar y mantener buenas relaciones con los actores locales; aspectos que no suelen tomar en cuenta los diseńadores de modelos de intervenciones para el desarrollo rural.

Por último, es importante mencionar la relevancia de las desigualdades y fracturas en las esferas locales. El hecho de que la asamblea comunitaria mantuviera una postura unitaria en las reuniones sos-tenidas con el personal de Kampo no quiere decir que todos los miembros de la asamblea coincidieran con las posturas sostenidas en el pleno. Durante los tres años que operó la iniciativa de Kampo en Nuevo Porvenir — de 2010 a 2013-, las personas con más poder, recursos y seguridad en la comunidad fueron adquiriendo una posición cada vez más crítica frente al programa y lograron construir cierto consenso en la asamblea en torno a sus perspectivas, imponiendo sus intereses, tanto en las decisiones de la asamblea como en relación con la asociación. 
Pero en la comunidad había quien se decía agradecido con Kampo y expresaba de forma privada su malestar frente al modo en que actuaban sus autoridades, recordando que todas las condiciones que estaban siendo cuestionadas habían sido aceptadas por cada uno de los integrantes del proyecto cuando se integraron a la iniciativa. Pero estas posturas raramente se expresaban de manera abierta, cualquier comentario que contradijera la posición de los líderes en los espacios colectivos de discusión se ignoraba, incluso los líderes llegaron a impedir que algunos miembros adelantaran parte de sus cuotas, acusando de "divisionistas" a quienes planteaban la conveniencia de permanecer en buenos términos con la asociación.

\section{Reflexiones finales}

Actualmente el microcrédito es considerado en muchos ámbitos como uno de los principales pilares para el desarrollo rural, y las instituciones de microfinanza se han convertido en actores centrales en el panorama financiero de muchos contextos rurales. La promoción de este tipo de estrategias se ha basado en los postulados que se desarrollan en la Teoría de la microfinanza, que identifica la exclusión de los servicios financieros como un elemento clave para explicar la pobreza rural, suponiendo que el microcrédito permite que los pequeños productores mantengan sus niveles de consumo a lo largo del proceso de producción, hagan inversiones para impulsar la producción agropecuaria de pequeńa escala y diversifiquen sus modos de sustento, mejorando así sus condiciones de vida y reduciendo su vulnerabilidad.

Este planteamiento resulta simplista e ignora el carácter complejo y multifactorial de la pobreza rural. El presente trabajo documenta algunos de los límites de la Teoría de la microfinanza a partir del análisis de una intervención de desarrollo rural estructurada a través de diversos proyectos de crédito; sumándose así a la literatura que en los últimos años ha cuestionado los supuestos teóricos en que se ha basado su difusión y la eficiencia de la microfinanza - y en particular del microcrédito- en la disminución de la pobreza.

El modelo de intervención que operaba en la comunidad de estudio compartía los postulados de la Teoría de la microfinanza, proponiendo 
una serie de pequeños créditos como estrategia para que un grupo de familias en pobreza extrema pudieran superar esta condición, desarrollando actividades económicas rentables e integrándose al mercado. Este proyecto es producto de las grandes expectativas que en los últimos años ha despertado la microfinanza en las instituciones internacionales de desarrollo, en algunos gobiernos, en organizaciones no gubernamentales y en muchos de los actores locales involucrados en estas iniciativas. Sin embargo múltiples experiencias han documentado que estas expectativas son difíciles de alcanzar.

Las familias de Nuevo Porvenir se integraron al proyecto propuesto por Kampo en un contexto que les ofrecía pocas alternativas de sustento, pero desde las primeras etapas de su operación se hizo evidente que no eran viables los planes de trabajo definidos por el personal operativo de esta asociación como condición para que fueran rentables los proyectos productivos. Las familias debían dedicar la mayor parte de su tiempo a asegurar su sobrevivencia cotidiana, los hombres salían a trabajar en las fincas y potreros de la región mientras las mujeres se ocupaban de las labores domésticas y la crianza de los niños. Si la familia había tenido que enfrentar algún gasto extraordinario, alguno de sus integrantes salía a trabajar algunos meses a la ciudad de México, y esto en muchos casos se traducía en el abandono temporal de los cultivos. Las capacidades de trabajo de las familias se veían con frecuencia afectadas cuando alguno de sus miembros se enfermaba. Además, en las tierras utilizadas para desarrollar los cultivos comerciales se sembraban también productos de autoabasto de los que dependía la alimentación básica de las familias, y esta asociación de cultivos limitaba la productividad agrícola. Las capacidades de inversión de estas familias eran muy reducidas y los créditos ofrecidos por el programa no cubrían todos los gastos requeridos para que sus plantaciones se desarrollaran de manera adecuada. No se contaban con medios de procesamiento y almacenamiento de las cosechas, por lo que los productores se veían obligados a vender sus productos a precios bajos impuestos por los intermediarios que pasaban a comprar los productos a la comunidad. Los integrantes de Nuevo Porvenir dudaban en integrarse en alternativas para vender sus productos directamente en el mercado regional, 
superando el control comercial de los rancheros locales, pues la subsistencia de sus familias dependía del trabajo que éstos les ofrecían en sus cafetales y pastizales. Además, para desarrollar alternativas de este tipo no bastaba el apoyo logístico ofrecido por Kampo, se requería tiempo y capacidades de gestión con actores regionales con las que no contaban los integrantes de la comunidad.

Frente al lugar preponderante que dieron las organizaciones internacionales de desarrollo a los proyectos de microcrédito como estrategias de desarrollo rural, el tema comenzó a ser cada vez más analizado y numerosos trabajos documentaron el fracaso de este tipo de iniciativas desde distintas perspectivas. Uno de los argumentos centrales de la Teoría de la microfinanza para explicar dicho fracaso es que los recursos destinados a la inversión productiva se integran a la lógica de la economía familiar, donde las inversiones compiten con las necesidades cotidianas del hogar. Pero éste no es el único problema que enfrenta la microfinanza en contextos rurales (Collins et al., 2011).

Diversos autores han señalado que las actividades agrícolas requieren inversiones importantes y dan rendimientos a mediano o largo plazo, por lo que resultan incompatibles con los pequeños créditos con cuotas de pago semanales o mensuales que ofrecen muchas de las iniciativas incluidas en programas de desarrollo rural (Doran et al., 2009; Marr, 2012; Taylor, 2012; Bateman, 2012). Estos argumentos han llevado a flexibilizar los esquemas utilizados por algunas instituciones de microfinanza y difundir servicios financieros paralelos al crédito, en particular el ahorro y los seguros (Montgomery, 1996; Marr, 2012).

El modelo de Kampo reconocía la necesidad de adaptar los términos de pago de los créditos de su programa a los ritmos y necesidades de la producción agrícola e integraba su esquema de créditos en una estrategia más amplia de desarrollo que incluía acciones de promoción de salud, educación y otras iniciativas que buscaban mejorar la calidad de vida de las familias. Sin embargo, este programa enfrentaba grandes retos pues su viabilidad dependía de la rentabilidad de las actividades agrícolas que promovía y su desarrollo se veía obstaculizado por múltiples circunstancias que rebasaban por mucho la esfera de su intervención. 
Este estudio muestra que las instituciones de microfinanza pueden adaptarse a las condiciones de la producción agropecuaria, pero no pueden cambiar aquellos factores de tipo estructural que limitan el desarrollo y reproducen la desigualdad. De manera más general, este trabajo busca mostrar algunos de los límites que enfrentan las intervenciones centradas en la esfera local, documentando el peso de factores contextuales en la reproducción de las condiciones de pobreza y desigualdad —entre los que destacan la marginación geográfica, la falta de infraestructura y la discriminación social (Shaw, 2004; Marr, 2012; Harper, 2012)_; intervenciones que tienen en el mejor de los casos efectos limitados (Banerjee et al., 2015) pero no pueden ser consideradas como motores de desarrollo que permitan superar las condiciones de pobreza, en ausencia de mecanismos que favorezcan la distribución de oportunidades y riqueza (Zeller et al., 1997; Shaw, 2004; Fouillet y Augsburg, 2008).

El material empírico recabado en el marco de la presente investigación también sugiere que las interacciones que se dan en torno a los distintos tipos de crédito son un espacio de dominación, pero también pueden ser un espacio de solidaridad, cuestionamiento y negociación en el que se hace evidente el carácter activo de los actores implicados en este tipo de proyectos.

Por último, es oportuno señalar que el uso del microcrédito en los programas de desarrollo rural es parte de una tendencia más general que se observa en las últimas décadas de transferir algunos de los costos asociados con las iniciativas de desarrollo a las esferas locales. El presente trabajo documenta cómo esta tendencia resulta problemática en contextos de pobreza extrema, donde los costos asociados con las intervenciones para el desarrollo rural corren el riesgo de entrar en competencia con las necesidades cotidianas de subsistencia de las familias y excluir a los sectores más vulnerables, que en el discurso de muchas de las agencias de desarrollo suelen ser identificados como beneficiarios prioritarios. 


\section{Bibliografía citada}

Ahlin, Christian y Robert Townsend, 2007, "Using Repayment Data to Test Across Models of Joint Liability Lending", Economic Journal, núm. 117, pp. 11-51.

Angulo Salazar, Lourdes del Carmen, 2004, "El acceso a crédito gubernamental para mujeres campesinas: ¿un derecho, una ayuda o una oportunidad?”, Magdalena Villarreal (coordinadora), Antropología de la deuda, Crédito, ahorro, fiado y prestado en las finanzas cotidianas, CIESAS, pp. 179-204.

Angulo Salazar, Lourdes del Carmen, 2014, "Prácticas financieras riesgosas para afrontar la crisis económica en los hogares: entre malabarismos con el dinero y sobreendeudamiento", Desacatos, núm. 44, pp. 51-66.

Armendáriz de Aghion, Beatriz y Jonathan Morduch, 2005, The Economics of Microfinance, The MIT Press.

Banerjee, Abhijit V., Timothy Besley y Timothy W. Guinnane, 1994, "Thy neighbour's keeper: The design of a credit cooperative with theory and a test", Quarterly Journal of Economics, vol. 109, núm. 2, pp. 491-515.

Banerjee, Abhijit, Dean Karlan y Jonathan Zinman, 2015, "Six Randomized Evaluations of Microcredit: Introduction and Further Steps", American Economic Journal: Applied Economics, vol. 7, núm. 1, pp. 1-21.

Bateman, Milford, 2012, "The Role of Microfinance in Contemporary Rural development Finance Policy and Practice: Imposing Neoliberalism as 'Best Price', Journal of Agrarian Change, vol. 12, núm. 4, pp. 587-600.

Bateman, Milford, Dean Sinković y Marinko Škare, 2014, "How to destroy an economy and community without really trying: The rise and fall of microcredit in post-conflict Bosnia", Ponencia presentada en el simposio Post-Crisis Recovery in South Eastern Europe: Policy Challenges for Social and Economic Inclusion, European Institute, London School of Economics, 27-28 marzo. 
Bateman, Milford y Ha-Joon Chang, 2009, "The Microfinance Illusion", Working Paper, University of Juraj Dobrila Pula, Croatia/University of Cambridge.

Besley, Timothy y Stephen Coate, 1993, "Group lending, repayment incentives and social collateral", Journal of Development Economics, núm. 46, pp. 1-18.

Bouquet, Emmanuelle, Betty Wampfler y Eliane Ralison, 2009, "Rice inventory credit in Madagascar: Conditions of access and diversity of rationales around an hybrid financial and marketing service", Rural Microfinance and Employment, Working Paper 2009-2, Rural Microfinance and Employment Protect, Institut de Recherche pour le Développement, en http://www.rumerural-micofinance.org

Cassar, Alessandra, Luke Crowley y Bruce Wydick. 2007, “The effect of social capital on group loan repayment: evidence from field experiments", The Economic Journal, núm. 117, pp. 85-106.

Chemin, Matthieu, 2008, "The benefits and costs of microfinance: evidence from Bangladesh", Journal of Development Studies, vol. 44, núm. 4, pp. 463-484.

Coleman, E. Brett, 1999, "The impact of group lending in Northeast Thailand ", Journal of development economies, núm. 60, pp. 105-141.

Collins, Daryl, Jonathan Morduch, Stuart Rutherford y Orlanda Ruthven, 2011, Las finanzas de los pobres. Cómo viven los pobres del mundo con dos dólares al día, Random House Mondadori [Título original: Portfolios of the Poor. How the World's Poor Live on \$2 a Day, 2009, Princeton University Press].

Czura, Kristina, 2015, "Pay, peek, punish? Repayment, information acquisition and punishment in a microcredit lab-in-the-field experiment", Journal of Development Economics, núm. 117, pp. 119-133.

Doran, Alan, Ntongi Mc Fadyen y Robert C. Vogel, 2009, The Missing Middle in Agricultural Finance, Relieving the capital constraint on smallholder groups and other agricultural SMEs, Oxfam GB Research Report. 
Duvendack, Maren, Richard Palmer-Jones, James G Copestake y Lee Hooper, 2011, What is the evidence of the impact of microfinance on the well-being of poor people?, EPPI-Centre, Social Science Research Unit, Institute of Education, University of London, London.

Fouillet, Cyril y Britta Augsburg, 2008, "La microfinance, un objet de pouvoir de plus? Conflit en Andhra Pradesh", Working Paper 2008-1, Rural Microfinance and Employment Proyect, Institut de Recherche pour le Développement, en http://www.rumerural-micofinance.org.

Fouillet, Cyril y Thierry Pairault, 2012, "Microfinanzas en China y La India: Una disciplina apropiada de forma diversa", en Magdalena Villarreal y Lourdes Angulo (coordinadoras), Las microfnanzas en los intersticios del desarrollo, Gobierno de Jalisco/Universidad Pedagógica Nacional Unidad Gdl./CIESAS/Secretaría de Promoción Económica, México.

Ghatak, Maitreesh, 1999, "Group lending, local information and peer selection", Journal of Development Economics, vol. 60, núm. 1, pp. $27-50$.

González de la Rocha, Mercedes (coordinadora), 2006, Procesos domésticos y vulnerabilidad. Perspectivas antropológicas de los hogares con Oportunidades, CIESAS, México.

Graeber, David, 2011, Debt. The first 5000 years, Melville House Publishing, Brooklyn, New York.

Griffin, Denis y Bryan W. Husted, 2015, "Social sanctions or social relations? Microfinance in Mexico", Journal of Business Research, núm. 68, pp. 2579 -2587. Guérin, Isabelle, 2011, "L'expérience vécue de la microfinance. Appropriations, tensions et recomposition des rapports sociaux", Cultures \& Conflits, núm. 83, pp. 39-56.

Guérin, Isabelle, 2015, La microfinance et ses dérives. Émanciper, discipliner ou exploiter?, Demopolis, Paris.

Guérin, Isabelle, Cyril Fouillet y Jane Palier, 2007, "La microfinance indienne peut-elle être solidaire?”, Revue Tiers Monde, núm. 190, pp. 291-308. 
Guérin, Isabelle; Marc Roesch, Santosh Kumar, Venkatasubramanian y Mariam Sangare, 2009, "Microfinance and the dynamics of financial vulnerability. Lessons from rural South India", Rural Microfinance and Employment Working Paper 20095, Rural Microfinance and Employment Project, Institut de Recherche pour le Développement, en http://www.rume-ruralmicofinance.org.

Guérin, Isabelle, Marc Roesch, Venkatasubramanian y Santosh Kumar, 2011, "The social meaning of over -indebtedness and creditworthiness in the context of poor rural South India households (Tamil Nadu)", Working Paper 2011-1, Rural Microfinance and Employment Project, Institut de Recherche pour le Développement, en http:// www.rume-rural-micofinance.org.

Harper, Marcom, 2012, "Microfinance Interest Rates and Client Returns”, Journal of Agrarian Change, vol. 12, núm. 4, pp. 564574.

Helth Lønborg, Onas y Ole Dahl Rasmussen, 2014, “Can Microfinance Reach the Poorest: Evidence from a Community-Managed Microfinance Intervention”, World Development, núm. 64, pp. 460-472.

Hermes, Niels y Robert Lensink, 2007, “The empirics of microfinance: what do we know?", The Economic Journal, núm. 117, Royal Economic Society, Oxford-Malden. Islam, Asadul, 2015, "Heterogeneous effects of microcredit: Evidence from large-scale programs in Bangladesh", Journal of Asian Economics, núm. 37, pp. 48.58.

Islam, Asadul, Chau Nguyen y Russel Smyth, 2015, "Does microfinance change informal lending in village economies? Evidence from Bangladesh”, Journal of Banking \& Finance, núm. 50, pp. 141-156.

Korht Marcel, Ruth Jacqueline Steward, Ruth Jacqueline Stewart, Carina Van Rooyen y Thea De Wet, 2012, "Microfinance: Development Intervention or Just Another Bank?”, Journal of Agrarian Change, vol. 12, núm. 4, pp. 575-586. 
Labie, Marc, Pierre-Guillaume Méon, Roy Mersland y Ariane Szafars, 2015, "Discrimination by microcredit officers: Theory and evidence on disability in Uganda", The Quarterly Review of Economics and Finance, núm. 58, pp. 44-55.

Long Norman, 2007, Sociología del desarrollo: una perspectiva centrada en el actor, CIESAS, México.

Marr, Ana, 2003, "A Challenge to the Orthodoxy Concerning Microfinance and Poverty Reduction", Journal of Microfinance, vol. 5, núm. 2, pp. 7-42.

Marr, Ana, 2012, "Effectiveness of Rural Microfinance: What We Know and What We Need to Know", Journal of Agrarian Change, vol. 12, núm. 4, pp. 555-563.

Montgomery, Richard, 1996, "Disciplining or protecting the poor? Avoiding the Social Cost of Peer Pressure in Micro -MicroCredit Schemes", Journal of International Development, vol. 8, núm. 2, pp. 289-305.

Mosley, Paul, 2001, "Attacking Poverty and The 'Post-Washington Consensus'”, Journal of International Development, núm. 13, pp. 307-313.

Mosley, Paul y David Hulme, 1998, "Microenterprise Finance: Is There a Conflict Between Growth and Poverty Alleviation?”, World Development, vol. 26, núm. 5, pp. 783-790.

Morvant-roux, Solène, 2009, "Accès au microcrédit et continuité des dynamiques d'endettement au Mexique: Combiner anthropologie économique et économétrie", Revue Tiers Monde, Armand Colin núm. 197, pp. 109-130.

Morvant-roux, Solène y Isabelle Guérin. 2012. "Los factores determinantes del uso de crédito: una reseña de la literatura sobre el tema”, en Magdalena Villarreal y Lourdes Angulo (coordinadoras), Las microfinanzas en los intersticios del desarrollo, Gobierno de Jalisco/Universidad Pedagógica Nacional Unidad Gdl./CIESAS/Secretaría de Promoción Económica, México.

Rajbanshi, Ram, Meng Huang y Bruce Wydick, 2015, “Measuring Microfinance: Assessing the Conflict between Practitioners and 
Researchers with Evidence from Nepal”, World Development, núm. 68, pp. 30-47.

Rankin, Katharine y Yogendra Shakya, 2012, "La política de la subversión en la práctica del desarrollo: una exploración de las microfinanzas en Nepal y Vietnam”, en Magdalena Villarreal y Lourdes Angulo (coordinadoras), Las microfinanzas en los intersticios del desarrollo, Gobierno de Jalisco/Universidad Pedagógica Nacional Unidad Gdl./CIESAS/Secretaría de Promoción Económica, México.

Rodríguez, Gerardo y Magdalena Villarreal, 2012, "Maromas y jineteos en las prácticas financieras de familias rurales en Ayuquila, Jalisco", Las microfinanzas en los intersticios del desarrollo. Cálculos, normatividades y malabarismos, CIESAS/FOJAL/UPN, Guadalajara, México.

Salas Casasola Ina, Denis Requier-Desjardins y Rita Schwentesius Rindermann, 2010, "Liberalización comercial agrícola y pobreza: una acentuación de las diferencias regionales", Gerardo Torres Salcido, Javier Sanz Cañada y José Muchnick (coordinadores), Territorios rurales. Pobreza, acción colectiva y multifuncionalidad, UNAM, México.

Schicks, Jessica, 2014, "Over-Indebtedness in Mocrifinance -An Empirical Analysis of Related Factors on the Borrower Level", World Development, núm. 54, pp. 301-324.

Sebstad, J. y M. Cohen, 2000, Microfinance, risk management and poverty, Washington DC, USAID AIMS Project.

Shaw, Judith, 2004, "Microenterprise Occupation and Poverty Reduction in Microfinance Programs: Evidence from Sri Lanka”, World Development, vol. 32, núm. 7, pp. 1247-1264.

Stiglitz, Joseph E., 1990, "Peer monitoring and credit markets", World Bank Economic Review, vol. 4, núm. 3, pp. 351-366.

Taylor, Marcus, 2012, “The Antinomies of 'Financial Inclusion': Debt, Distress and the Workings of Indian Microfinance", Journal of Agrarian Change, vol. 12, núm. 4, pp. 601-610.

Villarreal, Magdalena, 2000, "Deudas, drogas, fiado y prestado en las tiendas de abarrotes rurales", Desacatos, núm. 3, pp. 69-88. 
Villarreal, Magdalena, 2004, Antropología de la deuda. Crédito, ahorro, fiado y prestado en las finanzas cotidianas, Centro de Investigaciones y Estudios Superiores en Antropología Social, México.

Villarreal, Magdalena 2010. "Social Boundaries and Economic Dilemmas in Micro-financial Practices”, RUME Working Papers, Rural Microfinance and Employment Project, Institut de Recherche pour le Développement, IRD, Marseille.

Villarreal, Magdalena, 2014, "Presentación. De dineros, crisis y sobreendeudamientos", Desacatos, núm. 44, pp. 9-15.

Woolcock, Michael y Deepa Narayan, 2000, "Social Capital: Implications for Development Theory, Research, and Policy", World Bank Research Observer, vol. 15, núm. 2.

Zelizer, 1997, The social meaning of money, Princeton University Press. Zeller, Manfred, Aliou Diagne y Charles Mataya, 1997, "Market access by smallholder farmers in Malawi: Implications for technology adoption, agricultural productivity, and crop incom", Agricultural Economics, vol. 19, núm. 1-2, pp. 219-229 
